\title{
Interhospital Transfers: The Need for Standards
}

\author{
Kiran Gupta, MD ${ }^{1,2 *}$, Stephanie K. Mueller, MD, MPH'2,3
}

${ }^{1}$ Center for Clinical Excellence, Brigham and Women's Hospital, Boston, Massachusetts; 2 Harvard Medical School, Boston, Massachusetts; ${ }^{3}$ Division of General Internal Medicine, Brigham and Women's Hospital, Boston, Massachusetts.

Mrs. $S$ arrived to the medicine service at our hospital by ambulance transport at 9:00 PM. The intern on call received a page from the nurse, "Mrs. S has arrived. She is confused. Please assess." As is often the case, the intern had no prior knowledge of the patient's arrival, and review of medical records indicated that Mrs. S had never been seen at our hospital before.

The intern went to the bedside to assess the patient and found an elderly woman who appeared confused and was unable to provider her medical history, reason for the transfer, or details about her recent hospital course.

A few minutes later, the patient's son arrived at the bedside asking about her plan of care. The intern looked through the stack of papers in the envelope by her chart, and was able to locate reports of a recent chest $\mathrm{x}$-ray and abdominal computed tomography, as well as copies of brief progress notes, but was unable to find a transfer summary detailing her prior 5 days of hospitalization or reason for transfer. The patient's son was able to give some information, but he had just returned from a business trip and was not up to date on the details of his mother's hospital stay. Based on her son's input, the intern concluded the patient's somnolence was not her baseline; he performed an arterial blood gas and blood work, revealing profound acidemia and hyponatremia of unclear acuity. Mrs. S became hypotensive, requiring transfer to the intensive care unit. Several days later, she died.

This scenario highlights the potential dangers associated with patient transfers between acute care hospitals, known as interhospital transfer (IHT). Unfortunately, the described scenario is not a rare event. $^{1,2}$ Most providers who care for transferred patients can recount similar challenges when caring for IHT patients. ${ }^{3}$

Patient transfers from 1 hospital to another are common, affecting nearly 1 in 20 Medicare patients admitted to the intensive care unit ${ }^{4}$ and up to $50 \%$ of

\footnotetext{
*Address for correspondence and reprint requests: Kiran Gupta, MD, Center for Clinical Excellence, Brigham and Women's Hospital, 620 Tremont Street, Roxbury, MA 02120; Telephone: 617-732-8937;

Fax: 617-732-7072; E-mail: kgupta1@partners.org

Additional Supporting Information may be found in the online version of this article.

Received: September 14, 2014; Revised: December 18, 2014; Accepted: December 21, 2014

2015 Society of Hospital Medicine DOI 10.1002/jhm.2320

Published online in Wiley Online Library (Wileyonlinelibrary.com).
}

patients presenting with acute myocardial infarction, ${ }^{5}$ although reasons for transfer remain largely unstudied. The Emergency Medical Treatment and Active Labor Act requires a hospital to transfer patients who require a more specialized service unavailable at the subject institution, or when "medical benefits outweigh the increased risks to the individual." "Yet, this broad standard provides little guidance to clinicians in practice.

Identifying which patients may benefit from transfer is an ambiguous and subjective process. Studies show little agreement between the reasons cited for transfer among patients, transferring physicians, and receiving physicians, ${ }^{7}$ and incentives for transfer are often different between each stakeholder. For example, patients or families might initiate transfer for a second opinion from "a fresh set of eyes" because of a grim or uncertain prognosis or in the hope of a more promising or definitive medical opinion. Similarly, referring physicians may initiate transfer for particular procedures, surgeries, or consultations that the receiving physician may ultimately decide will be of little clinical benefit to the patient. Such heightened expectations and changes to the care plan as agreed on by the patient and referring physician may affect the patient's perceptions of care at the receiving institution, although exactly how remains unknown. Alternatively, patients and families may desire transfer because of previously established relationships with providers at another institution, or they may be dissatisfied with certain aspects of care at the referring institution. Referring institutions may initiate transfer for a variety of reasons, including inability to provide a needed procedure or test, patient/family preference, or protocol, among others. Receiving hospitals usually have an interest in maintaining a large referral base for the sake of both revenue and reputation, but may also view accepting transfers as part of their larger mission to provide expert consultation and specialty services that may not be available at the referring institution. Additional proposed benefits include strengthening provider networks, promoting clinical diversity, and improving the educational experience of trainees often present at the accepting institution. Although patients, providers, and referring and accepting hospitals all undoubtedly benefit from various aspects of the IHT, further research is needed to more clearly identify which patients are most likely to benefit from transfer and why. 
Once the decision to transfer/accept a patient has been made, there are no clear guidelines over how this process should be executed. For this reason, care providers at community hospitals describe IHT as "frustrating" and "time consuming." 8 Referring providers may face challenges identifying an accepting hospital due to the limited capacity of the receiving institution, reaching the correct receiving physician, and managing delays in transfer once the patient is accepted. ${ }^{8}$ Similarly, accepting physicians may be frustrated by the time waste associated with accepting a patient that ends up transferred to another facility, limited authority to triage the patient to the most appropriate accepting service, inability to predict time of patient arrival, and missing pieces of critical information at time of patient arrival, among other reasons. Furthermore, incompatible electronic health records make access to data from the referring institution difficult. For example, without standards for transferring imaging, patients may undergo unnecessary and costly duplicate imaging leading to delay in needed procedures. Existing guidelines are largely focused on equipment and expertise required for the physical transfer of the patient, but fail to consider other aspects of the transfer process that may be critical for patient safety such as protocols for communication of patient information and transfer of completed imaging. As such, hospitals are largely left to devise their own protocols for IHT, which often differ between hospitals as well as between different services within 1 hospital. ${ }^{1,3}$

Although it is true that many patients benefit from IHT, the process introduces inherent vulnerability into healthcare delivery. Moving a patient between facilities exposes that individual to risks associated with discontinuity of care, well described in the literature on intrahospital patient handoffs (ie, the transfer of patient care responsibility from 1 provider to another within 1 hospital), which can lead to excessive costs and poor patient outcomes. ${ }^{9}$ Presumably, such risks are even greater for patients transferred between hospitals than for those transferred between providers within 1 hospital, because system factors like electronic health records, nursing and ancillary staff continuity, and accessibility of transferring provider are not in place to mitigate communication gaps. Furthermore, unlike discharges home or to subacute care facilities, also known to be error prone and lead to adverse events, ${ }^{10,11}$ in the case of IHT, patients are often more acutely ill and less stable. In fact, limited data suggest that aside from a select subset of patients requiring specialized care, individuals transferred may have increased resource utilization and greater-thanexpected mortality than those who are not transferred. ${ }^{1,2,12}$ Moreover, these findings may not be entirely attributable to medical complexity among transferred patients.

Today, the process of IHT varies tremendously across US hospitals, ${ }^{1}$ differences that may have significant implications for both cost and patient safety out- comes. Standardization of IHT, including patient selection and information exchange between transferring and accepting providers/institutions, is imperative to improve the quality and safety of this process. As demonstrated with other common, high-risk care transitions, such as intrahospital patient handoffs and patient discharge, creating basic guidelines of practice (such as including important data elements at time of care transfer) ${ }^{13,14}$ is necessary to improve quality of the care transition.

However, to achieve high-quality standardization, we must first methodologically conduct rigorous clinical research to understand fundamental issues of the IHT process, including why patients are transferred (from the perspective of patients and transferring and accepting institutions), which patients benefit most from transfer and why, and how various IHT processes impact health outcomes. Interventions such as communication and data transfer tools, feedback mechanisms between referring and accepting institutions, and other evidence-based guidelines can then be designed to improve IHT based on the findings of this research while still allowing for flexibility of individual patient needs. Additional work is then needed to implement and rigorously evaluate the effects of such interventions on patient and provider outcomes including, but not limited to, length of stay, adverse events, mortality, readmissions, and patient satisfaction measures. In summary, by focusing research and quality improvement initiatives on these vital questions, we can begin to improve the quality of care we provide to patients during this critical transition of care.

Disclosure: Nothing to report.

\section{References}

1. Sokol-Hessner L, White A, Feldman K, Hohmann S. Patients transferred from outside hospitals to academic hospitalists and general internists have higher mortality and costs than patients from the ED. Paper presented at: Society of Hospital Medicine National Conference; May 2013; Washington, DC.

2. Hernandez-Boussard T, Davies S, McDonald K, Wang NE. Interhospital facility transfers in the united states: a nationwide outcomes study [published online ahead of print November 13, 2014]. J Patient Saf. doi: 10.1097/PTS.0000000000000148.

3. Mueller SK, Schnipper JL. Physician perspectives on inter-hospital transfers. Paper presented at: Society of Hospital Medicine National Conference; March 2014; Las Vegas, NV.

4. Iwashyna TJ, Christie JD, Moody J, Kahn JM, Asch DA. The structure of critical care transfer networks. Med Care. 2009;47(7):787-793.

5. Iwashyna TJ, Kahn JM, Hayward RA, Nallamothu BK. Interhospital transfers among Medicare beneficiaries admitted for acute myocardial infarction at nonrevascularization hospitals. Circ Cardiovasc Qual Outcomes. 2010;3(5):468-475.

6. U.S. House of Representatives. Office of the Law Revision Counsel. Examination and treatment for emergency medical conditions and women in labor. Title 42 USC $\$ 1395$ dd. Available at: http://www. gpo.gov/fdsys/granule/USCODE-2010-title42/USCODE-2010-title42chap7-subchapXVIII-partE-sec1395dd. Accessed October 292014.

7. Wagner J, Iwashyna TJ, Kahn JM. Reasons underlying interhospital transfers to an academic medical intensive care unit. J Crit Care. 2013;28(2):202-208.

8. Bosk EA, Veinot T, Iwashyna TJ. Which patients and where: a qualitative study of patient transfers from community hospitals. Med Care. 2011;49(6):592-598.

9. Lawrence RH, Tomolo AM, Garlisi AP, Aron DC. Conceptualizing handover strategies at change of shift in the emergency department: a grounded theory study. BMC Health Serv Res. 2008;8:256. 
10. Forster AJ, Murff HJ, Peterson JF, Gandhi TK, Bates DW. The incidence and severity of adverse events affecting patients after discharge from the hospital. Ann Intern Med. 2003;138(3):161-167.

11. Walz SE, Smith M, Cox E, Sattin J, Kind AJ. Pending laboratory tests and the hospital discharge summary in patients discharged to subacute care. J Gen Intern Med. 2011;26(4):393-398.

12. Bernard AM, Hayward RA, Rosevear J, Chun H, McMahon LF. Comparing the hospitalizations of transfer and non-transfer patients in an academic medical center. Acad Med. 1996;71(3): 262-266.

13. Starmer AJ, Spector ND, Srivastava R, Allen AD, Landrigan CP, Sectish TC. I-pass, a mnemonic to standardize verbal handoffs. Pediatrics. 2012;129(2):201-204.

14. Hospital Medicine Reengineering Network (HOMERUN) Collaborative. Executive summary. Available at: https:/members.aamc.org/ eweb/upload/HOMERUN\%20summary\%202012.pdf. Accessed July 23, 2013. 